\title{
ESTUDIOS SOCIO-JURÍDICOS
}

Bogotá, D. C. • enero-junio de 2020

Volumen 22 - Número 1

ISSN 0124-0579 • ISSNe 2145-4531

Dol: http://dx.doi.org/10.12804/revistas.urosario.edu.co/sociojuridicos/v221

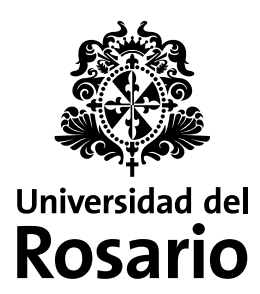

IB-Índice bibliográfico. Clarivate: SciELO Citation Index; IBN-Publindex de Colciencias (B).

BBCS-Bases bibliográficas con comité de selección. Research Data bases -EBSCO: Fuente Académica Plus, Fuente Académica Premier; SciELO Colombia; Red de Revistas Científicas de América Latina y El Caribe; España y Portugal - RedALyC; Citas Latinoamericanas en Ciencias Sociales y Humanidades-CLASE; Dialnet de la Universidad de la Rioja; Red Iberoamericana de Innovación y Conocimiento científico -REDIB; Directory of Open Access Journals DOAJ; Clasificación Integrada de Revistas Científicas-CIRC (Ciencias sociales-C).

Bases de datos bibliográficas. Latindex de la Universidad Nacional Autónoma de México -UNAM-; Plataforma BIBLAT de la UNAM; Google Scholar; Ulrich's International Periodicals Directory; Scilit; European Reference Index for the Humanities -ERIH; Electronic Journals Library (Elektronische Zeitschriftenbibliothek o EZB); Vlex; MIAR; Actualidad Iberoamericana. 


\section{PRESENTACIÓN}

La revista Estudios Socio-Jurídicos de la Facultad de Jurisprudencia de la Universidad del Rosario es una publicación científica arbitrada que presenta semestralmente a la comunidad académica y a la sociedad en general los resultados de investigación en las áreas de teoría jurídica, sociología jurídica, antropología jurídica y las tradicionales del campo jurídico (derecho público, derecho privado, derecho penal, derechos humanos y derecho internacional) desde perspectivas interdisciplinarias.

RECTOR

José Alejandro Chayne García

VICERRECTORA

Stéphanie Lavaux

CONSILIARIOS

Carolina Durán, Alberto Fergusson, Merlín Grueso, Andrés López, Ángel Melguizo

SÍNDICO

Miguel Francisco Diago Arbeláez

SECRETARIA GENERAL

Catalina Lleras-Figueroa

COMITÉ CIENTÍFICO

Dennis Rodgers, Universidad de Amsterdam, Países Bajos; Allan BrewerCarias, Universidad Central de Venezuela, Venezuela; Clara Sandoval, Essex University,

Reino Unido; Camilo Rodríguez Yong,

Universidad del Rosario, Colombia;

Diego Rodríguez-Pinzón, American University, EE. UU.

\section{COMITÉ EDITORIAL}

Alberto Cerda, Universidad de Chile, Chile; Manuel Alcántara-Sáez, Universidad de Salamanca, España; Álvaro Vidal-

Olivares, Pontificia Universidad Católica de

Valparaíso, Chile; Manuel Alberto Restrepo, Universidad del Rosario, Colombia; Manuel Fernando Quinche, Universidad del Rosario, Colombia; Ómar Darío Heffes, Universidad de La Plata, Argentina; Roberto Vidal-López, Pontificia Universidad Javeriana, Colombia; Liliana ObregónTarazona, Universidad de los Andes, Colombia.
DECANO

José Alberto Gaitán Martínez

VICEDECANA

Laura Victoria García Matamoros

EDITORA

Rocío del Pilar Peña Huertas

ASISTENTE EDITORIAL

Laura Duperret Gómez

COLABORACIÓN ESPECIAL

María Nilsa Africano Fonseca

VOLUMEN 22

NÚMERO 1: ENERO-JUNIO DE 2020

ISSN: 0124-0579 - ISSNE: 2145-4531

DOI DEL NÚMERO:

http://dx.doi.org/10.12804/revistas.urosario. edu.co/sociojuridicos/v221

\section{PUBLICACIONES PERIÓDICAS}

Tatiana Morales Perdomo

Editorial Universidad del Rosario

CORRECCIÓN DE ESTILO

Mónica Quinana Rey

DIAGRAMACIÓN Y ARMADA

Precolombi EU-David Reyes

IMPRESIÓN Y ENCUADERNACIÓN

Xpress. Estudio Gráfico y Digital SAS

CORRESPONDENCIA

Carrera 6 \# 15-18

Edificio Jockey Club - Oficina 8

esj@urosario.edu.co,

IMPRESO EN COLOMBIA

PRINTED IN COLOMBIA

Las opiniones de los artículos solo comprometen a los autores y en ningún caso al Consejo Editorial ni a la Universidad del Rosario. Se permite la reproducción total o parcial de los artículos citando la fuente.

Visítenos en la internet: http://revistas.urosario.edu.co/sociojuridicos 


\section{Contenido}

DOI DEL NÚMERO: http://dx.doi.org/10.12804/revistas.urosario.edu.co/sociojuridicos/v221

Editorial

\section{Autor extranjero invitado}

Cómo debemos entender el concepto de "patrón de violencia

política": repertorio, objetivo, frecuencia y técnica

What Should we Mean by "Pattern of Political Violence"?

Repertoire, Targeting, Frequency and Technique

Como devemos entender o conceito de "Padrão de Violência

Política": repertório, objetivo, frequência e técnica

FRANCISCO GUTIÉRREZ-SANÍN

ELISABETH JEAN WOOD

La violación como práctica de guerra: hacia una tipología

de la violencia política

Rape as a War Practice: Toward a Typology of Political Violence

O estupro como prática de guerra: para uma tipologia da violência política ELISABETH JEAN WOOD

\section{Artículos de investigación}

La Rebelión de la Prisión de Attica (Nueva York, 1971): opresión racial, encarcelamiento en masa y la retórica de la igualdad

The Attica Prison Uprising (New York, 1971): Racial Oppression,

Mass Incarceration and the Rhetoric of Equality

A rebelião da Prisão de Attica (Nova York, 1971): opressão racial,

encarceramento em massa e a retórica da igualdade

EVANDRO PIZA DUARTE

Marcos Vinícius Lustosa QueIroz

RAFAel De Deus García 
La aceptabilidad de las acciones wrongful birth en el marco de la Teoría Discursiva del Derecho de Jürgen Habermas

The Acceptability of Wrongful Birth Actions within Jürgen

Habermas' Discursive Theory of Law

A aceitabilidade das ações wrongful birth no marco da Teoria

Discursiva do Direito de Jürgen Habermas

LUIS GUILLERMO BASTIDAs GOYES

EDUARDO ALFONSO RUEDA BARRERA

Caracterización de la violencia de pareja en el contexto

de la minería aurífera en dos municipios de influencia

del páramo de Santurbán, Colombia

Intimate Partner Violence Characteristics in Two Gold Mining

Towns in Santurbán, Colombia between 2008-2015

Caracterização da violência na relação de casal no contexto

da mineração aurífera em dois municípios de influência

do páramo de Santurbán, Colômbia

ReNÉ Álvarez Orozco

LUCÍA ANDRADE MANJARRÉS

Yinny Paola Valencia Atuesta

Liberación de la madre tierra: entre la legitimidad

y los usos sociales de la ilegalidad

Liberation of Mother Earth: Between the Legitimacy

and the Social Uses of Illegality

Liberação da mãe terra: entre a legitimidade e os usos

sociais da ilegalidade

BRYAN VARGAS REYES

ROSEMBERT ARIZA SANTAMARÍA

Reinserción social de adolescentes en conflicto con la ley.

Un estudio conceptual

Social Reintegration of Juveniles in Conflict with the Law.

A Conceptual Study

Reinserção social de adolescentes en conflito com a lei.

Un estudo conceitual

LIZETH MONTEjANO TORRES

JaIme Sebastián F. Galán JimÉneZ

Paola Iliana de la Rosa Rodríguez 
Corte Suprema y participación ciudadana: reflexiones

a partir de una audiencia pública de la Corte argentina

Supreme Court and Public Participation: Reflections from

a Public Hearing in the Argentine Court

Corte Suprema e participação cidadã: reflexões a partir

de uma audiência pública da Corte argentina

JIMENA SÁENZ

LETICIA BARRERA

Límites a la práctica de compensación bancaria en los contratos

de depósito de cuenta de ahorros en modalidad de nómina como garantía de derechos mínimos de los consumidores financieros categorizados como 'trabajadores'.

Un análisis de derecho colombiano

Bank Clearing Limits on Payroll Savings Account Agreement

as a Guarantee of Minimum Rights of the Financial Consumers

Categorized as 'Workers'. A Colombian Law Analysis

Limites à prática de compensação bancária nos contratos

de depósito de conta poupança em modalidade de nómina como garantia de direitos mínimos dos consumidores financeiros categorizados como 'trabalhadores'. Uma análise de direito colombiano ANGIE LORENA RINCÓN HIGUERA

Otros sujetos de derecho o personas (?)

Other Subjects of Law or People (?)

Outros sujeitos de direito ou pessoas (?)

Franco Ceballos Rosero

El precedente administrativo en Colombia:

implicaciones y dificultades

The Administrative Precedent in Colombia: Implications and Difficulties

O precedente administrativo na Colômbia; implicações e dificuldades

RICARDO QUECÁN GAMBA 
Entre la juridificación y la profundización de la democracia social: elementos para pensar los conflictos alrededor de los manuales de convivencia en las instituciones educativas y los derechos fundamentales de los estudiantes

Between Juridification and Deepening Social Democracy: Key

Elements to Discuss the Conflicts Around the Rules of Coexistence within Educational Institutions and the Fundamental Rights of Students

Entre a juridificação e o aprofundamento da democracia social: elementos para pensar os conflitos ao redor dos manuais de convivência nas instituições educativas e os direitos fundamentais dos estudantes JaVier Orlando AguirRe Román ANa Patricia Pabón Mantilla IVEth Paola Moreno Bernal

\section{Reseñas}

García Arboleda, J.F. (Ed.) (2017). Psicoanálisis y Derecho.

Bogotá: Siglo del Hombre Editores

Dekker, H. A. L. (2017). The Invisible Line: Land Reform, Land Tenure Security and Land Registration.

London: Routledge

BRyan TRIANA ANCINEZ

Akiola, A., \& Wissink, H. (2019). Trajectory of Land Reform in Post-Colonial African States. Springer International Publishing. 